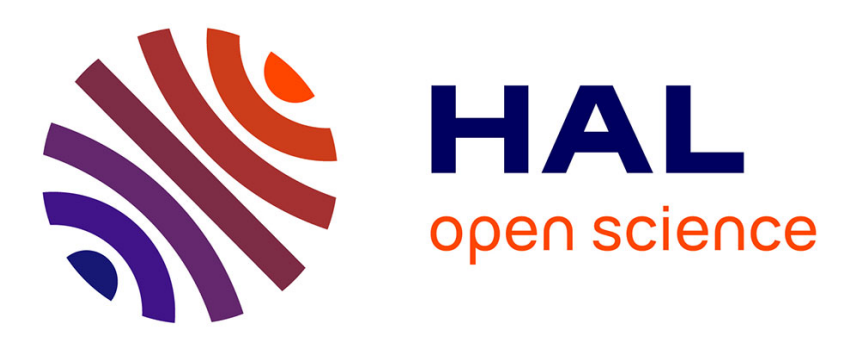

\title{
Permanence et variation des unités prosodiques dans le discours et l'interaction
}

Cristel Portes, Roxane Bertrand

\section{To cite this version:}

Cristel Portes, Roxane Bertrand. Permanence et variation des unités prosodiques dans le discours et l'interaction. Journal of French Language Studies, 2011, pp.97-110. hal-00576851

\section{HAL Id: hal-00576851 \\ https://hal.science/hal-00576851}

Submitted on 15 Mar 2011

HAL is a multi-disciplinary open access archive for the deposit and dissemination of scientific research documents, whether they are published or not. The documents may come from teaching and research institutions in France or abroad, or from public or private research centers.
L'archive ouverte pluridisciplinaire HAL, est destinée au dépôt et à la diffusion de documents scientifiques de niveau recherche, publiés ou non, émanant des établissements d'enseignement et de recherche français ou étrangers, des laboratoires publics ou privés. 
Article paru dans Journal of French Language Studies, volume 21, issue 01, pp. 97-110. doi:10.1017/S0959269510000499

La version définitive est disponible en ligne à l'adresse suivante :

http://journals.cambridge.org/action/displayAbstract?fromPage=online \&aid=8002965 \&fulltextType=RA\&fileId=S0959269510000499

\title{
Permanence et variation des unités prosodiques dans le discours et l'interaction
}

Cristel Portes et Roxane Bertrand

Laboratoire Parole et Langage

\begin{abstract}
Our aim is to show that the units defined in the laboratory by intonational phonology are not only identifiable in 'naturally occurring data' but also play a structuring role in discourse and interaction. However, in order to achieve their identification, one must disentangle the phonological structure they build from other types of information that receive prosodic encoding. Such include both 'orthogonal' and gradient prosodic variations of pitch range and tempo that encode, for instance, information structure or reported speech, and 'disfluencies', mostly due to speech production processes, which play an important role in turn taking negotiation.
\end{abstract}

\section{Introduction}

On admet couramment dans la littérature qu'il est difficile de retrouver dans des corpus de parole spontanée les unités prosodiques définies par la phonologie intonative, voire que la parole spontanée connaît une structuration qui lui est spécifique. C'est ainsi qu'on oppose l'approche 'grammaticale' de l'intonation à une approche où l'intonation est conçue comme un 'marqueur de contextualisation' sur la base du type de données qu'elles choisissent, respectivement élicitées ou spontanées (Couper-Kuhlen, 2001 : 14-17). Notre but ici est de 
montrer qu'il est non seulement possible d'identifier dans le discours spontané les unités de la phonologie intonative, mais encore que ces unités jouent un rôle organisateur pour le discours dans l'interaction.

La difficulté provient de la complexité des données 'naturelles' qui conjoignent des informations discursives et interactionnelles, plus contrôlées en laboratoire. En effet, les discours attestés mettent en jeu des relations de natures diverses entre les énoncés (relatives, par exemple, à la structure informationnelle, à l'organisation des thèmes discursifs ou simplement des relations de cohérence discursive) qui ne sont pas spécifiquement élicitées sous le contrôle du linguiste. Alors que les données de laboratoire sont le plus souvent monologales, les données 'spontanées' mettent en présence plusieurs interlocuteurs en interaction qui hésitent, s'ajustent les uns aux autres, expriment émotions et attitudes, citent, imitent, endossent différents rôles, etc., toutes choses qui influencent la mise en forme prosodique des énoncés qui les transmettent. Nous pensons que ces différentes contraintes commencent à être suffisamment connues et décrites pour êtres séparées les unes des autres de telle sorte que les unités phonologiques puissent être plus aisément retrouvées sous l'accumulation des marques.

Dans cet article, nous nous proposons de poser les principales distinctions qui permettent de séparer, dans des données non contrôlées, la structure phonologique des autres types d'information, discursives, interactionnelles et de planification, encodées par la prosodie. A partir de données du français soumises à notre expertise par les éditeurs de ce volume, nous établirons les éléments définitoires des unités phonologiques dans la section 1 en les distinguant de deux types de faits interférant avec elles : d'une part, ce que Di Cristo et al., (2004) nomment 'les variations prosodiques orthogonales', présentées dans la section 2 ; d'autre part les 'disfluences' que nous traiterons dans la section 3. Nous défendons ainsi une approche unifiée dans laquelle une grammaire commune à la lecture et à la conversation met 
en forme simultanément des informations de natures diverses dont les traces s'entremêlent dans la parole.

\section{Identifier les unités phonologiques}

Ce n'est pas le propos du présent article de discuter les nombreux modèles existants de la structure intonative du français. Nous présenterons plutôt quelques propriétés empiriques consensuelles qui peuvent être utilisées pour identifier les constituants phonologiques et quelques problèmes empiriques qui font parfois obstacle à cette identification.

\subsection{Propriétés de la structure intonative du français}

L'intonation du français est généralement décrite comme comportant deux niveaux de constituance : un domaine mineur et un domaine majeur (Di Cristo, 1999 ; Jun \& Fougeron, 2000, 2002 ; Post, 2000).

Le domaine mineur est caractérisé par deux événements tonals: un accent final obligatoire et un mouvement montant initial optionnel. L'accent final est essentiellement marqué par un mouvement tonal notable et l'allongement de la syllabe précédant la frontière du constituant. Il est associé à la dernière syllabe pleine du constituant (à l'exclusion des syllabes dont le noyau est un schwa). Le mouvement montant initial (dont le statut accentuel est en débat) se synchronise le plus souvent avec l'une des premières syllabes du premier mot lexical du constituant mineur.

Le domaine majeur, souvent assimilé au syntagme intonatif de la phonologie prosodique, est caractérisé par un allongement final plus important que celui du domaine mineur et par la présence de tons de frontière. En français, ces derniers se placent le plus souvent sur la même syllabe que les accents finals, ce qui rend leur identification respective plus difficile que dans d'autres langues. Toutefois, comme dans d'autres langues, la 
combinaison des accents mélodiques et des tons de frontière définissent différents contours mélodiques qui véhiculent des instructions pragmatiques et interactionnelles distinctes.

Un contour majeur subordonne un ou plusieurs domaines mineurs comme le montre la figure 1 : en haut, la figure 1a) montre un domaine majeur contenant 3 domaines mineurs dans le corpus INFORDIA de conversation informelle, alors que la figure 1b) montre un domaine majeur à 4 domaines mineurs provenant du corpus FORLEC de lecture. Notez que, dans ce dernier exemple, les unités phonologiques contiennent un plus grand nombre de mots que dans l'extrait 1b. On observe une assez grande variabilité de longueur des unités phonologiques qui semble dépendre en partie du genre de discours mais aussi du type de séquence discursive à l'intérieur d'un même genre. Ce point est relativement peu explorée (voir toutefois Degand et Simon, 2009: 13-14). Cette variabilité peut faire obstacle à l'identification précise des unités phonologiques en opérant des regroupements non prédits par les contraintes syntaxiques et prosodiques. 


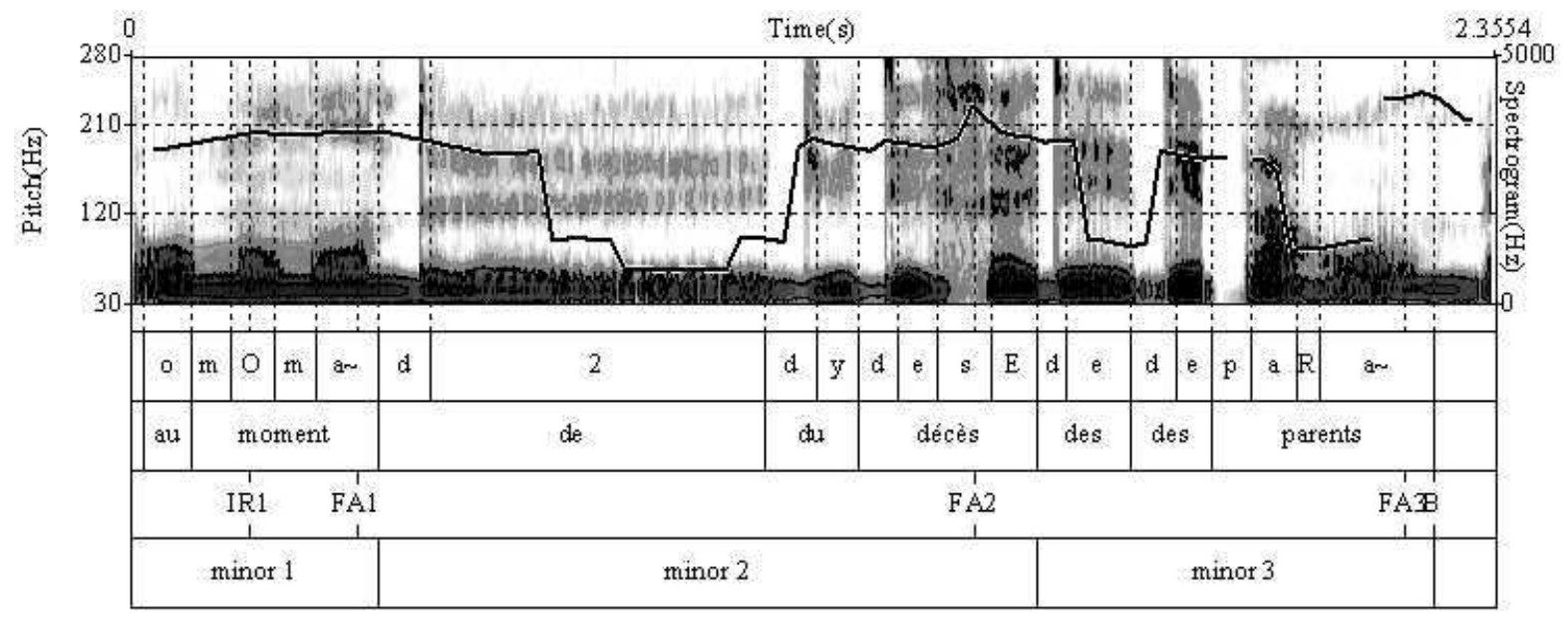

a)

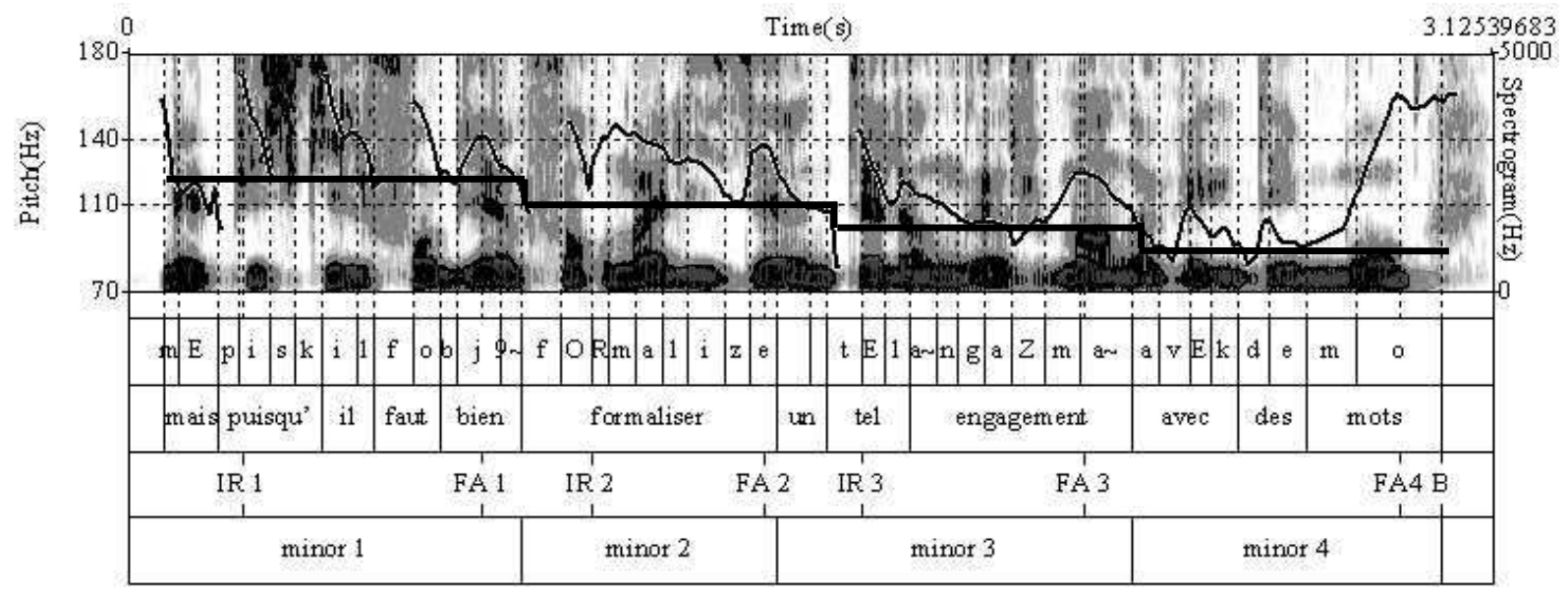

b)

Figure 1. Une unité majeure regroupant des unités mineures: a) dans la conversation, b) dans la lecture. 'IR' = montée initiale et ' $F A$ ' = accent final. La ligne noire en escalier représente l'abaissement régulier (downstep) des domaines mineurs à l'intérieur du domaine majeur.

\subsection{Identifier les domaines mineurs}

Il existe une certaine variabilité de réalisation des domaines mineurs selon le nombre, la qualité et la position des événements tonals qu'ils contiennent. Par exemple, Jun et Fougeron (2000, 2002) distinguent six réalisations de surface différentes pour leur 'syntagme accentuel'. Une grande partie de cette variabilité peut être expliquée par le caractère facultatif 
de la montée initiale. Ainsi, dans la figure 1, les constituants mineurs 'minor 1' de a) et 'minor 1, 2 et 3' de b) contiennent à la fois une montée initiale (IR) et un accent final (FA) alors que les constituants 'minor 2 et 3' de 1) et 'minor 4' de b) ne présentent pas de montée initiale.

De ce fait, et quel que soit le type de données (lues ou spontanées), il peut être difficile de décider si un événement tonal doit être considéré comme une montée initiale (IR) ou comme un accent final (FA) et donc d'identifier l'empan d'un domaine mineur. Cela s'avère particulièrement délicat lorsque le mot lexical est monosyllabique ou dissyllabique comme dans la figure 2: sur les mots 'place', 'banque' et 'prends' les contraintes syntaxiques prédisent la réalisation d'un accent final FA marquant la frontière droite d'un domaine mineur car ces mots sont les têtes de leurs syntagmes respectifs (Post, $2000: 34$ ). Mais par ailleurs, le FA sur 'Hugo' est réalisé plus haut et sur une syllabe plus allongée que le mouvement mélodique précédent sur 'place', ce qui suggère d'identifier ce dernier à une montée initiale. La relation entre le mouvement sur 'banque' (IR) et celui sur 'angle' (FA) nous semble reproduire la structure précédente par symétrie. Dans de tels cas d'ambiguïté, une meilleure connaissance des détails phonétiques fins d'implémentation des catégories telles que l'allongement de l'attaque ou de la coda syllabique ou des différences d'alignement du mouvement tonal avec les unités segmentales, par exemple, permettraient sans doute de trancher entre IR et FA. 


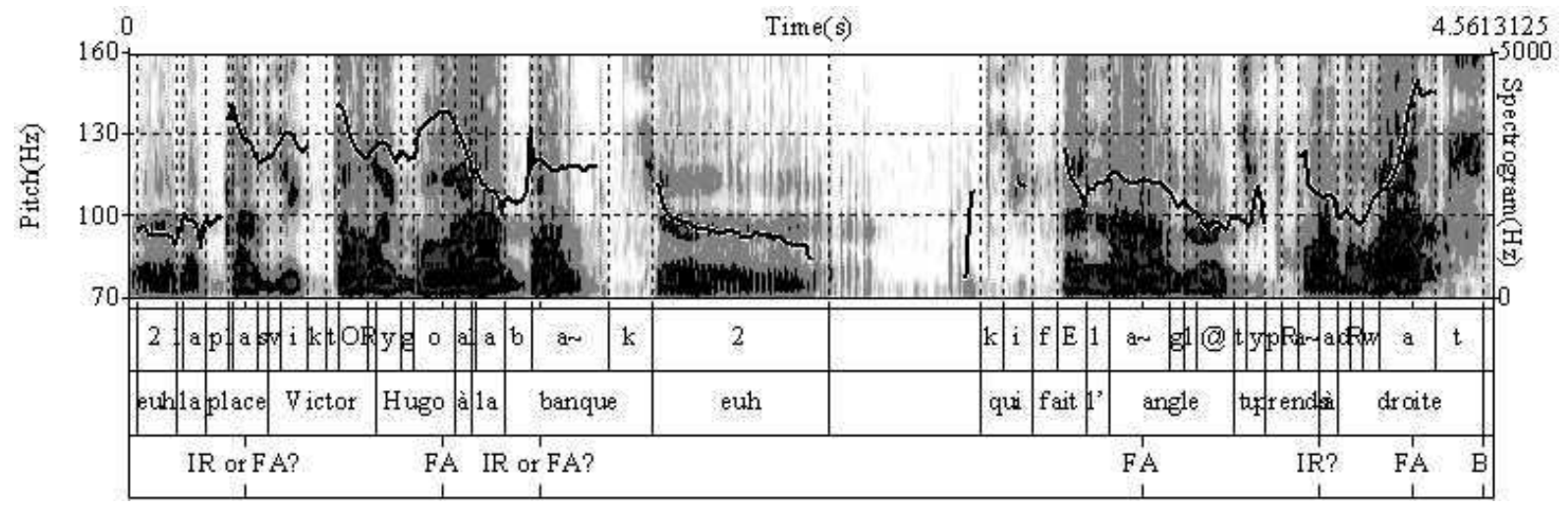

Figure 2. Extrait du corpus INFORITI montrant un domaine majeur pour lequel il est difficile de décider combien de domaines mineurs il contient. Les mots monosyllabiques 'place' et 'banque' présentent un événement tonal difficile à classer en IR ou FA.

\subsection{Identifier les domaines majeurs}

On identifie les domaines majeurs d'abord par la force de leurs frontières, qui se réalisent avec un allongement plus marqué que celui des domaines mineurs, mais aussi par le contour intonatif qu'ils portent. La littérature sur l'intonation du français décrit trois ou quatre contours de base (voir Marandin, 2006 pour une synthèse récente) : un contour descendant régulièrement associé aux notions de finalité (de séquence topicale, de tour) ou de certitude (le locuteur transmet qu'il n'anticipe aucune révision de la part de l'interlocuteur); un contour montant associé aux notions de continuité, d'interrogation ou d'incertitude (le locuteur transmet qu'il anticipe une révision possible du contenu) ; un mouvement montant-descendant dit aussi parfois 'd'implication' (Delattre 1966) associé à la notion d'engagement du locuteur. Un quatrième contour montant-descendant depuis la syllabe pénultième est parfois décrit comme une variante du précédent, notamment parce qu'il transmet aussi l'engagement du locuteur avec cette nuance que convaincre sera plus difficile ou plus conflictuel.

L'identification des domaines majeurs peut s'appuyer sur une troisième caractéristique : le fait que les domaines mineurs qui les composent se réalisent fréquemment sur des paliers 
d'abaissement successifs (downstep). L'abaissement des domaines mineurs est clairement visible sur la figure 1b) où il est interrompu par le contour de continuation final. Ce type d'effet est généralement conçu soit comme une tendance globale appelée 'déclinaison de phrase' dans les modèle dits 'superpositionnels' ou comme la récurrence de changements de registre locaux dans les modèles que Ladd (1996) nomme 'downstep models'. Berg et al. (1992) ont proposé de modéliser le 'downstep' comme une ligne de référence définie par les cibles tonales hautes $(\mathrm{H})$. Toutefois, dans la figure $1 \mathrm{~b})$, l'abaissement des domaines mineurs est mieux mis en évidence par l'intermédiaire d'une ligne de référence définie à partir des cibles tonales basses (L) : cette observation est cohérente avec la proposition de Ladd de concevoir les cibles basses comme représentant plus rigoureusement le niveau du registre à cause de leur plus grande stabilité (voir Ladd, 1996 et la section 2 plus loin).

\subsection{Le 'paraton'}

De nombreuses études empiriques sur des données variées ont mis en évidence le marquage prosodique d'unités discursives souvent comparées aux paragraphes dans le discours écrit. Sur des données spontanées, Brown \& Yule (1983) définissent le 'paraton' essentiellement à partir de traits de frontière comme une fréquence particulièrement haute en début de séquence et la présence d'un allongement particulièrement significatif en fin de séquence, d'un abaissement dans le bas du registre tonal et de pauses silencieuses dépassant 200 millisecondes. Sluijter \& Terken (1993) ont trouvé un abaissement régulier des phrases successives dans des paragraphes de parole lue élicitée en laboratoire. Ces paragraphes intonatifs sont souvent associés à des segments de discours thématiquement cohérents entre lesquels on a pu observer des remises à niveau du registre tonal. Il est dès lors pertinent d'ajouter une unité phonologique supplémentaire à notre inventaire, définie notamment par l'abaissement des domaines majeurs qui l'accompagne fréquemment. De tels paragraphes 
intonatifs sont très communs dans le corpus FORLEC où ils se terminent souvent par un contour descendant jusqu'aux valeurs les plus basses du registre du locuteur comme attendu en lecture. Toutefois, des séquences d'abaissement d'unités majeures peuvent aussi se terminer par des contours montants ainsi qu'on l'observe dans des données plus interactionnelles (corpus INFORDIA). Dans de tels cas, l'abaissement des unités majeures s'avère un indice plus fiable du paraton (ou d'une séquence définie en tours de parole comme le récit, voir ci-après) que les indices de frontières.

\subsection{Les unités phonologiques construisent les tours de parole}

Dans le cadre de l'analyse conversationnelle (Sacks et al. 1974), les tours de parole sont construits à partir d'unités de construction des tours (turn-constructional units, désormais TCU). Depuis l'étude de Ford et Thompson (1996), on sait que le critère intonatif joue un rôle tout aussi crucial que le critère syntaxique pour déterminer un TCU. Selting (2000) définit un TCU final comme un point de complétion possible de l'unité qui rend possible une alternance de tour. Un point (ou place) pertinent de transition (transition-relevance place, désormais TRP) apparaît donc comme un point d'achèvement potentiel déterminé à la fois par des indices syntaxiques, pragmatiques et intonatifs. Ces différents indices sont utilisés par les auditeurs comme des indices de prédictibilité. Les caractéristiques du domaine intonatif majeur (décrites ci-dessus) sont donc utilisées par les partenaires de l'échange pour projeter, reporter et reconnaître les TRP. Les contours intonatifs associés à la fin du domaine intonatif majeur, par le sens qu'ils véhiculent (cf section 1.3), créent des attentes spécifiques qui nécessitent des réponses adaptées. En produisant un contour intonatif conclusif (descendant ou montant terminal), le locuteur signale à son partenaire une place potentielle de transition. A l'inverse, les contours montants de continuation se révèlent les indices les plus pertinents pour projeter une suite et favoriser le report de la TRP à la fin d'une séquence comme le récit 
par exemple, qui implique la production de plusieurs TCU non finaux avant d'atteindre son point d'achèvement (Selting, 2000). Conjointement aux indices syntaxiques, les contours intonatifs montants peuvent aussi jouer ce rôle dans les structures à deux composants (Lerner, 1991) telles que 'si ... alors', et caractériser la fin de la première partie de l'unité syntaxique. La figure 1 illustre un cas de ce type (énoncé introduit par 'puisqu'il') dans le corpus de lecture qui permet de mettre en évidence la pertinence de ces notions de projection dans des corpus de données plus contrôlées.

L'interlocuteur joue un rôle dans le processus de production des tours de parole (Fox Tree, 1999) en émettant des signaux appelés 'backchannels' (désormais BC) ${ }^{1}$ qui ponctuent l'apparition des unités phonologiques. Ils apparaissent de manière significative après les domaines intonatifs majeurs caractérisés par un contour montant continuatif (Bertrand et al, 2007). L'usage d'un tel contour permet au locuteur de s'assurer de l'écoute de son partenaire tout en confortant son rôle de locuteur principal ; en produisant un $\mathrm{BC}$, l'interlocuteur prouve alors qu'il reconnaît ces espaces ménagés par le locuteur tout en satisfaisant ses attentes. Certains BC, plus fréquemment mimo-gestuels que vocaux, peuvent également apparaître après des unités mineures où ils assument plutôt des fonctions 'de relance' : l'interlocuteur assure au locuteur principal qu'il lui laisse le tour (malgré le vide ponctuel dans son discours en cas de recherche de mot par exemple-), ou anticipe son adhésion à ce qui va être produit tout en l'encourageant ainsi à poursuivre (Portes et Bertrand, 2006). L'exemple 1 illustre un cas dans lequel la locutrice principale $\mathrm{N}$ perçoit l'adhésion et l'encouragement de son interlocutrice L; elle rebondit alors sur ce dernier non pas pour continuer mais au contraire pour laisser L poursuivre à sa place :

Exemple $1^{2}$

\footnotetext{
${ }^{1}$ On connaît surtout leur rôle dans le processus de compréhension des discours en tant que signaux manifestant l'écoute et la compréhension du discours courant.

${ }^{2}$ Nous utilisons le / pour marquer le domaine mineur et le // pour le domaine majeur.
} 


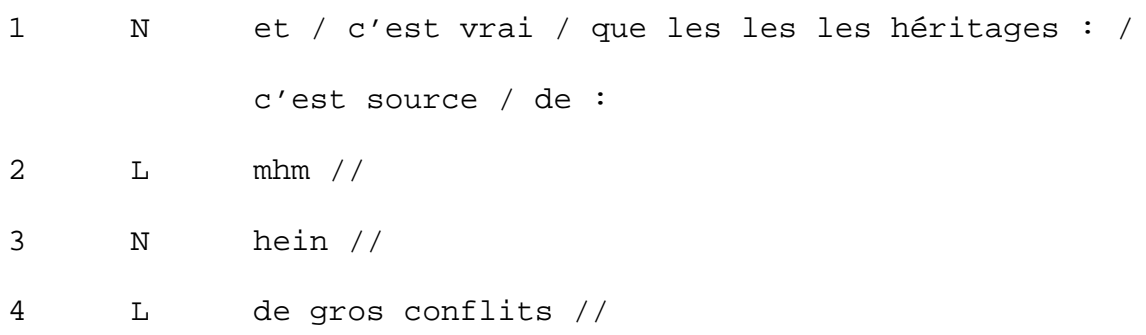

\section{Les variations prosodiques orthogonales}

La première proposition du présent article consiste à insister sur l'importance de séparer très clairement les aspects de l'implémentation prosodique qui relèvent de la réalisation des unités phonologiques discrètes des variations prosodiques graduelles qui peuvent s'appliquer par ailleurs sur ces unités pour transmettre des significations pragmatiques et interactionnelles supplémentaires.

Di Cristo et al. (2004) conçoivent ces variations comme les dimensions orthogonales des trois modules de l'organisation prosodique qu'ils décrivent : la variation de la force des proéminences dans l'organisation métrique, la variation du registre tonal dans l'organisation tonale et la variation du tempo dans l'organisation temporelle. Par ailleurs, Gussenhoven (2004) évoque le rôle dans la production des significations paralinguistiques de trois 'codes biologiques' (le code de la fréquence, le code de l'effort et le code de production) qui contrôlent l'implémentation phonétique. Ils peuvent jouer indépendamment de la volonté du locuteur ou au contraire être manipulés intentionnellement dans des buts pragmatiques.

Nous reviendrons ici sur deux aspects des variations orthogonales qui jouent un rôle pragmatique et interactionnel important et sont susceptibles d'ajouter leurs indices à ceux qui signalent la structure prosodique : les variations du tempo et les variations du registre tonal.

\subsection{Variations du tempo}


La partie métrique du rythme relève de la structure phonologique qu'elle contribue à construire. En revanche, la dimension graduelle du 'tempo' ou débit de parole varie indépendamment, en suivant des influences contextuelles ou interactionnelles (Auer et al., 1999).

Le tempo peut être mesuré très simplement en comptant le nombre de syllabes ou de mots par unité de temps (seconde ou minute) pour une séquence donnée. Par ailleurs, plusieurs faits prosodiques contribuent à l'organisation temporelle de la parole : durée des syllabes, types de pauses (silencieuses ou remplies). Parmi ces pauses, certaines sont produites intentionnellement par le locuteur pour réaliser différents objectifs pragmatiques ('pauses pragmatiques') alors que d'autres émergent comme le produit accidentel de l'activité d'encodage (pauses d'encodage). Même si la frontière entre ces deux types de pauses n'est pas toujours très claire, nous parlerons des pauses pragmatiques dans la présente section et nous réserverons la question des pauses d'encodage pour la section 3, dévolue aux disfluences.

L'insertion de pauses silencieuses est très fréquente dans les styles à la rhétorique très contrôlée comme en témoignent les discours politiques ou pédagogiques où être clairement compris et convaincre sont des enjeux majeurs (Duez, 1999). La figure 3, extraite du corpus FORDIA, illustre le ralentissement du tempo dans un discours à valeur pédagogique réalisé grâce à l'insertion de pauses silencieuses. 


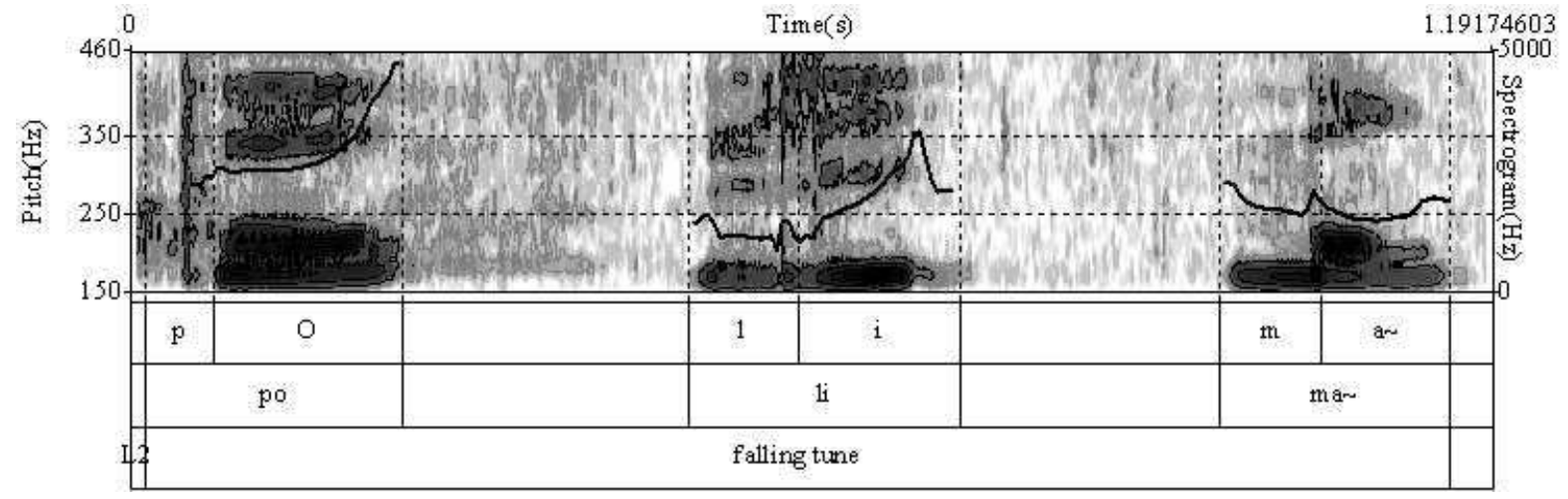

Figure 3. Le mot 'poliment' est asserté seul dans un domaine majeur, chacune de ses syllabes étant réalisée dans son propre domaine mineur. Des pauses silencieuses sont insérées entre chaque syllabe.

L'énoncé de la figure 3, qui contient un unique mot asserté seul dans un domaine majeur, transmet le conseil que donne une mère à son fils de demander quelque chose 'poliment'. Le locuteur produit chaque syllabe avec une proéminence métrique, un allongement et un accent mélodique (clairement montant sur les deux premières syllabes), ce qui a pour conséquence d'affecter un domaine mineur à chacune. En outre, le locuteur insère deux pauses inattendues à l'intérieur du mot, séparant les trois syllabes les unes des autres. Malgré cela, le contour majeur descendant jusqu'au milieu du registre qui s'applique sur le mot entier (c'est-à-dire sur le domaine majeur) est reconnu sans difficulté par l'interprète comme transmettant une mise en garde légèrement menaçante. Le phrasé séparé des syllabes et l'insertion des pauses qui provoquent un ralentissement notable du débit transmettent les nuances d'insistance et d'irritation avec une force qui varie graduellement en fonction de la proportion du ralentissement.

Dans cet exemple, le ralentissement du tempo affecte la réalisation des unités phonologiques dans la mesure où il conduit au phrasé séparé des syllabes d'un même mot, normalement liées. Cela n'empêche pas l'analyste d'identifier trois domaines mineurs qui composent un domaine majeur porteur d'un contour intonatif interprétable. 


\subsection{Variations du registre tonal}

De même que les variations du tempo interfèrent avec le marquage de la structure phonologique à travers l'allongement des syllabes, les variations graduelles du registre tonal interfèrent avec la différence relative des tons hauts et bas en fonction de leur hauteur d'implémentation. Pourtant, une montée ou une chute intonatives restent une montée ou une chute même si ces mouvements sont produits plus haut dans le registre fréquentiel du locuteur. Ils devraient aussi rester reconnaissables et surtout différents l'un de l'autre même si la plage de fréquence disponible devient plus étroite ou plus large. Ladd (1996) propose de modéliser les variations du registre tonal en distinguant deux dimensions de variation indépendantes : 1) le 'niveau du registre' correspond à la hauteur globale à laquelle une séquence est énoncée : selon Ladd, la meilleure mesure pour cette dimension correspond à la moyenne des valeurs des cibles basses qui sont les plus stables ; 2) 'l'étendue du registre' correspond à la plage fréquentielle utilisée dans une séquence : on la mesure en soustrayant la moyenne des valeurs des cibles basses à celle des cibles hautes.

Les variations du registre tonal ne sont pas seulement le signal de l'identité du locuteur (par exemple de son âge ou de son sexe), elles peuvent aussi être manipulées intentionnellement pour réaliser différents objectifs communicatifs comme signaler le focus attentionnel de l'énoncé ou changer le statut d'une séquence (signaler un épisode de discours rapporté, par exemple), entre autres (Wichmann, 2000).

Il a été très clairement montré pour de nombreuses langues dont le français que la structure informationnelle a un impact sur la hauteur d'implémentation de la séquence qu'elle distingue : les constituants 'donnés' sont énoncés plus bas et plus compressés que les constituants 'nouveaux' (voir Breen et al., à paraître, pour une revue récente). La figure 4 
montre que l'information d'arrière-plan peut être produite plus bas alors que l'information spécifiquement assertée est réalisée globalement plus haut et avec une étendue plus large.

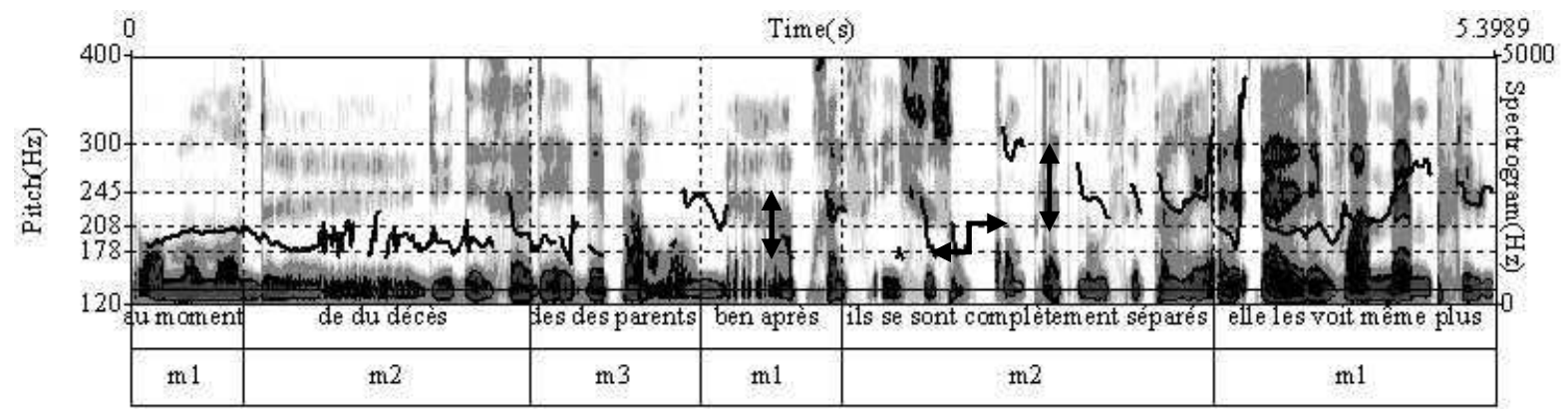

Figure 4. Extrait du corpus INFORDIA. La seconde partie de l'énoncé, qui correspond au focus informationnel, est réalisée avec un niveau plus élevé (flèches horizontales) et une étendue plus large (flèches verticales) que la première partie correspondant à une information d'arrière-plan.

Remarquez que, dans cet exemple, le changement de registre commence avec la réalisation d'une montée initiale emphatique sur la première syllabe de 'complètement'. Cela s'explique par le fait qu'en français, la montée initiale contribue très souvent au marquage de la frontière gauche du domaine focal (Di Cristo 1999, Beyssade et al to appear). Remarquez également que malgré les variations du registre tonal, les domaines prosodiques majeurs et mineurs sont clairement reconnaissables, notamment par l'intermédiaire de l'allongement préfinal lorsque l'étendue du registre est très compressée (Di Cristo \& Jankowsky, 1999).

\section{Les disfluences}

Les disfluences se révèlent une caractéristique incontournable de la parole spontanée, ce qui a fait émerger une perspective de recherche qui vise précisément à caractériser leurs indices lexicaux, morphologiques ou acoustico-prosodiques (Carlson et al., 2006). Leur modélisation 
au niveau acoustique permet de mieux comprendre leur rôle et les difficultés éventuelles qu'elles posent dans la compréhension des discours en raison notamment des 'interruptions' qu'elles introduisent dans le flux de parole (Schriberg 1994).

On admettra ici que les disfluences sont les traces de l'activité d'encodage, dues pour l'essentiel à la simultanéité de la conceptualisation et de la production. Le terme de disfluence réfère à des phénomènes d'auto-correction, aux constituants inachevés (pour certains appelés 'amorces'), à certaines répétitions d'items et à certaines pauses silencieuses ou remplies. Il s'agit de distinguer clairement les répétitions et les pauses silencieuses disfluentes de celles qui sont produites intentionnellement par le locuteur à des fins pragmatiques (cas de l'insistance pour des répétitions comme 'c'est très très très ennuyeux'; ou encore les 'pauses pragmatiques' évoquées en section 2.1).

Bien que les traces de l'encodage ne soient pas intentionnelles, il importe toutefois de les étudier et de les modéliser non seulement pour les distinguer des faits pragmatiques évoqués ci-dessus, mais aussi pour montrer comment elles interfèrent avec la structuration du message.

Dans l'exemple 2 ci-dessous, les éléments disfluents que sont une longue pause remplie (euh) suivie d'une pause silencieuse, rendent l'identification du domaine mineur qu'elles interrompent, plus difficile (voir figure 2). Cette identification est grandement facilitée si on modélise clairement la disfluence comme un phénomène distinct et interférant.

\section{Exemple 2}

(de) la place Victor Hugo/ à la banque euh \# qui fait l'angle/ tu prends à droite//

Modéliser les disfluences et les séparer de la structuration du message ne signifie pas les éliminer mais au contraire leur donner leur juste place. Cela permet par exemple de rendre 
compte des fonctions qu'elles assument dans l'interaction, notamment pour signaler un point potentiel de transition de tour.

Les énoncés inachevés (d'un point de vue syntaxique) sont souvent considérés comme des cas d'énoncés disfluents. Il nous paraît fondamental de considérer que les unités prosodiques, au même titre que les unités syntaxiques ou sémantiques, peuvent rester inachevées. Mais cet argument n'est viable que dans la mesure où l'on défend, comme ici, une conception phonologique de la structure intonative.

Les énoncés inachevés peuvent aussi être complétés, modifiés, c'est-à-dire réparés par le locuteur ou l'interlocuteur. Ce dernier cas est illustré dans l'exemple 1 (section 1.5) qui met en évidence le principe crucial de coopération inhérent à toute conversation, et la mise en œuvre incessante, pour le satisfaire, de phénomènes d'alignement inter-locuteurs. Dans une étude récente, Chevalier et Clift (2008) s'intéressent à ces énoncés inachevés qui sont révélateurs, pour ces auteurs, d'une difficulté ou d'un problème dans le discours du locuteur. Ne pas compléter un tour apparaît alors comme une manière de rechercher une certaine 'affiliation' avec son interlocuteur. L'exemple 1 est une parfaite illustration de ce point. L'unité intonative amorcée par $\mathrm{N}$ (ligne 1) est en ce sens complétée et achevée par l'interlocutrice L (ligne 4). Nous constatons que l'intégrité des unités phonologiques est totalement préservée si on admet de transcender le seul discours du locuteur.

Nous conclurons sur cet exemple qui soulève une autre difficulté pour la détermination des unités : non seulement co-produite par les deux locutrices, l'unité considérée est en outre scindée par le mini-échange (2-3) relatif à la négociation des tours, ce qui en fait une unité à la fois co-construite et discontinue.

\section{Conclusion}


Dans cet article, nous avons montré la pertinence des unités phonologiques que pose l'approche grammaticale pour la description de différents types de corpus, spontanés et conversationnels aussi bien que lus. L'analyse de données très contrôlées permet notamment d'isoler les contraintes propres au système phonologique qu'il importe de ne pas attribuer indûment à des influences externes : par exemple, on a montré qu'en français, le nombre de syllabes du mot lexical influence la réalisation d'une montée initiale dans le constituant mineur. Une telle contrainte phonologique s'applique évidemment aux données spontanées où elle entre en interaction avec d'autres contraintes sur la réalisation de la montée initiale, par exemple le marquage de la frontière gauche du focus informationnel. Nous avons montré que si la conjonction des marquages prosodiques de faits linguistiques et pragmatiques dans la parole spontanée pouvait compliquer leur identification respective, une séparation théorique bien définie entre structure intonative, variations prosodiques graduelles et faits de performance (disfluences) permettait d'y voir plus clair. Réciproquement, défendre une conception phonologique (grammaticale, abstraite) des unités prosodiques, quel que soit le type de données, permet de comprendre et d'expliquer comment ces unités, lors de leur implémentation en parole, adaptent leur réalisation phonétique à la co-occurrence d'autres phénomènes, par exemple l'interruption par une pause d'encodage ou un changement de tour lors de la co-énonciation, sans perdre leur intégrité.

\section{References}

Auer, P. , Couper-Kuhlen, E. \& Müller, F. (1999). Language in Time : The Rhythm and Tempo of Spoken Interaction. Oxford : Oxford University Press.

Beyssade, C. ; Hemforth, B. ; Marandin, J.-M. \& Portes (to appear). Prosodic Markings of Information Focus in French. Proceedings of the $3 d$ conference 'Interface Discours Prosodie'. Paris, 9-11 september 2009. 
Berg, R. van den, C. Gussenhoven \& A. Rietveld (1992). Downstep in Dutch: Implications for a model, Gerard J. Docherty \& D. Robert Ladd (eds) Papers in Laboratory Phonology II: Gesture, Segment, Prosody. Cambridge: Cambridge University Press. 335-359.

Bertrand, R.; Ferré, G.; Blache, P.; Espesser, R.; Rauzy, S. (2007) Backchannels revisited from a multimodal perspective, Proceedings of Auditory-visual Speech Processing Hilvarenbeek, The Netherlands, p. 1-5. Cederom.

Bertrand, R.; Portes, C.; Sabio, F. (2007) Distribution syntaxique, discursive et interactionnelle des contours intonatifs du français dans un corpus de conversation. Travaux Neuchâtelois de Linguistique, 47, p. 59-77. ISSN 1010-1705.

Breen, M., Fedorenko, E., Wagner, M., Gibson, E., (under revision). Acoustic correlates of information structure. Language and Cognitive Processes.

Brown, G. \& Yule, G. (1983). Discourse analysis. Cambridge University Press

Carlson R., Gustafson K., Strangert E. (2006): "Cues for hesitation in speech synthesis", In INTERSPEECH-2006, paper 1516-Tue3BuP.2.

Chevalier F. H.G. \& Clift R. (2008) Unfinished turns in French conversation: Projectability, syntax and action, Journal of Pragmatics, Volume 40, issue $10 \mathrm{p}$. 1731-1752., ISSN: 0378-2166.

Couper-Kuhlen, E. (2001) Intonation and Discourse : Current Views from within. In Schiffrin, Tannen Hamilton (eds), The handbook of Discourse Analysis, Blackwell.

Degand, L. and Simon, A.-C. (2009). On identifying basic discourse units in speech: theoretical and empirical issues, Discours, 4 | 2009, (on line paper) URL : http://discours.revues.org/index5852.html.

Delattre P., (1966) Les dix intonations de base du français, French review 40 : 1-14. 
Di Cristo, A. (1999). Le cadre accentuel du français contemporain, Langues 3(2), pp.184205, Langues 4(2), pp. 258-267.

Di Cristo, A \& Jankowski L. (1999). Prosodic organization and phrasing after focus in French. Proceedings of XIVth International Congress of Phonetics.

Di Cristo, A., C. Auran, R. Bertrand, C. Chanet, C. Portes (2004). Outils prosodiques et analyse du discours. Cahiers de l'Institut de Linguistique de Louvain, vol. 28. p. 27-84.

Duez, D.(1999). La fonction symbolique des pauses dans la parole de l'homme politique. Faits de langues, vol. 13., p. 91-97.

Ford C. and Thompson S. (1996) "Interactional units in conversation: Syntactic, intonational and pragmatic resources for the management of turns," in Interaction and Grammar, E. Ochs, E. Schegloff, and S. Thompson, Eds. Cambridge, pp. $134-184$.

Fox Tree, J. E. (1999). Listening in on monologues and dialogues. Discourse Processes, $27,35-53$

Gussenhoven C. (2004). The phonology of tone and intonation. Cambridge University Press.

Harvey S.; Schegloff E.A.; Jefferson G. (1974) A Simplest Systematics for the Organization of Turn-Taking for Conversation, Language, Vol. 50, 4, pp. 696-735.

Jun, S.-A., \& Fougeron, C. (2000). A phonological model of French intonation. In A. Botinis (ed.), Intonation: Analysis, modelling and technology. Boston: Kluwer.

Jun, S.-A., \& Fougeron, C. (2002). Realizations of accentual phrase in French. Probus, 14, $147-172$.

Kern F. (2007), Prosody as a resource in children's game explanations: Some aspects of turn construction and recipiency, Journal of Pragmatics 39 (2007) 111-133.

Ladd (1996). Intonational phonology. Cambridge University Press. 
Lerner G. (1991) On the syntax of sentences-in-progress, Language in Society 20, 441458.

Marandin, J-M. (2006): 'Contours as Constructions'. In: Schoenefeld, D. (guest ed.): Constructions all over: case studies and theoretical implications. DIPP (digital peer publishing) NRW. http://www.constructions-online.de/articles/specvol1/

Portes C. and Bertrand R. (2006) Some cues about the interactional value of the 'continuation' contour in French. In: Auran, C. et al. (eds.): Discours \& Prosody as a Complex Interface (IDP06), (Cederom 14p.).

Post, Brechtje (2000). Tonal and phrasal structures in French intonation. The Hague: Holland Academic Graphics.

Schriberg, E. (1994). Preliminaries to a Theory of Speech Disfluencies. Doctoral dissertation. University of California, Berkeley.

Selting, M. (2000): 'The construction of 'units' in conversational talk', Language in Society, 29: 477-517.

Wichmann, A. (2000). Intonation in Text and Discourse: beginnings, middles and ends. Pearson education. 